\title{
The Representative Porcine Model for Human Cardiovascular Disease
}

\author{
Yoriyasu Suzuki, ${ }^{1}$ Alan C. Yeung, ${ }^{2}$ and Fumiaki Ikeno ${ }^{2}$ \\ ${ }^{1}$ Division of Cardiovascular Medicine, Nagoya Heart Center, 461-0045 Aichi, Japan \\ ${ }^{2}$ Division of Cardiovascular Medicine, School of Medicine, Stanford University, 300 Pasteur Drive, Falk CVRB007, Stanford,
} CA94305, USA

Correspondence should be addressed to Fumiaki Ikeno, fikeno@stanford.edu

Received 17 September 2010; Accepted 13 December 2010

Academic Editor: Andrea Vecchione

Copyright (๑) 2011 Yoriyasu Suzuki et al. This is an open access article distributed under the Creative Commons Attribution License, which permits unrestricted use, distribution, and reproduction in any medium, provided the original work is properly cited.

To improve human health, scientific discoveries must be translated into practical applications. Inherent in the development of these technologies is the role of preclinical testing using animal models. Although significant insight into the molecular and cellular basis has come from small animal models, significant differences exist with regard to cardiovascular characteristics between these models and humans. Therefore, large animal models are essential to develop the discoveries from murine models into clinical therapies and interventions. This paper will provide an overview of the more frequently used large animal models, especially porcine models for preclinical studies.

\section{Introduction}

Therapies for the treatment of cardiovascular disease have advanced dramatically over the last 2 decades. To improve human health, scientific discoveries and technologies must be translated into practical applications. Such advances typically begin with basic research and then progress to the clinical level. Inherent in the development of these technologies is the role of preclinical testing using animal models. Although no animal model can fully replicate the complexity of human pathological conditions, animal models are key for the evaluation of mechanisms of disease and testing of diagnostic technologies and interventions. Significant insight into the molecular and cellular basis of cardiovascular biology has come from small animal models, particularly mice. However, significant differences exist with regard to cardiovascular characteristics, when mice are compared to humans. Therefore, large animal models that approximate human physiology, function, and anatomy, are essential to develop discoveries from murine models into clinical therapies and interventions.

This paper will provide an overview of the more frequently used large animal models, especially porcine models for preclinical studies.

\section{Experimental Porcine Model of Myocardial Ischemia}

Recent years have seen 2 important changes to the clinical approach involving acute myocardial infarction (AMI). First, there is increasing recognition that the pathophysiology of human AMI is a process occurring at many levels, not just within the epicardial coronary artery, but also within the microvasculature and the myocardium. Second, contemporary treatments are shifting from thrombolytic dissolution of epicardial coronary thrombus to direct mechanical approaches such as angioplasty and stents. On the other hand, chronic reversible contractile dysfunction (hibernation) is frequently identified in the evaluation of patients with coronary artery disease and constitutes an intense area of clinical interest. Thus, the following discussion reviews and examines current animal models of $\mathrm{AMI} /$ chronic hibernation and discusses advantages and limitations, providing an overview of their place in these important models of AMI.

2.1. Animal Myocardial Infarction/Reperfusion Model. Traditionally, in vivo large animal studies have involved dogs 


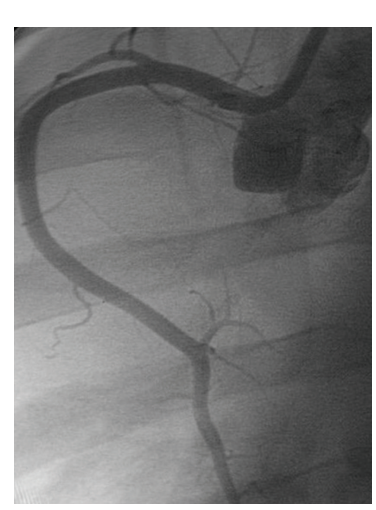

(a)

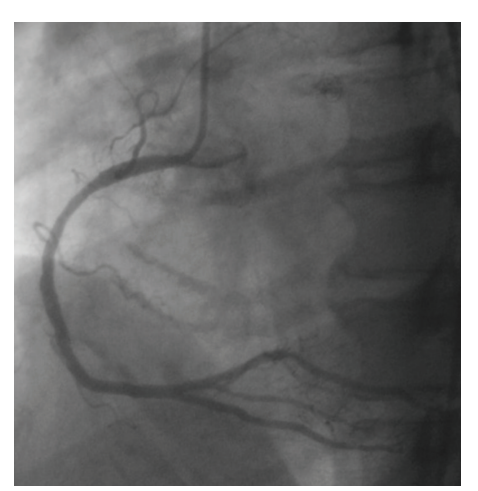

(c)

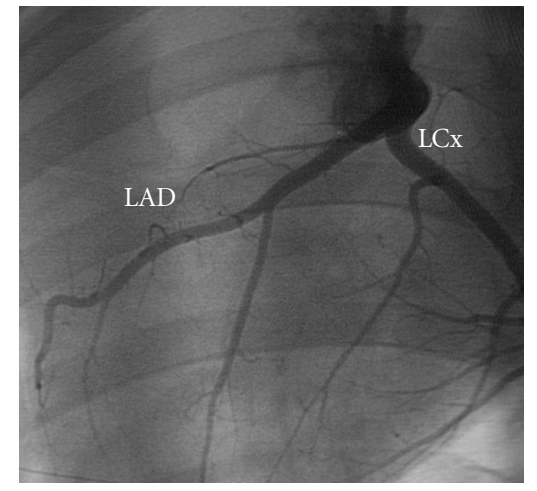

(b)

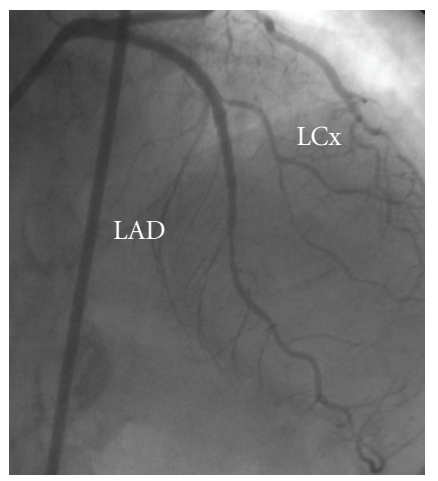

(d)

Figure 1: Porcine (a and b). (a) Right coronary artery. (b) Left coronary system. Human (c and d). (c) Right coronary artery. (d) Left coronary system. Similar anatomy and coronary distribution is shown of the left anterior descending, left circumflex, and right coronary arteries.

as the most frequently used animal species yet due to cost and social pressures, the porcine model has become a more favored animal only in recent years $[1,2]$. To induce myocardial ischemia, whereas the left descending coronary artery (LAD) is most frequently used to produce regional myocardial ischemia, left circumflex coronary artery (LCx) occlusions have also been used. A number of studies have been performed in open-chest models with thoracotomy [3, 4]. This approach allows easy access to the heart with direct visual control of the success of coronary artery occlusion and the opportunity to measure local contractile performance, metabolic parameters, and coronary flow using ultrasound techniques [5-7]. However, disadvantages of this open-chest model stem from having to open the chest and pericardium, both of which are suspected and reported to influence the pattern of left ventricular remodeling in chronic models $[8,9]$. To eradicate these disadvantages, several groups have reported catheter-based closed-chest techniques [9-12]. Cardiac catheterization and coronary intervention in the pig and in humans are similar in many ways (Figure 1). Through the use of an intracoronary balloon inflation technique, both the location and duration of coronary artery occlusion are well controlled. In our laboratory, once baseline coronary angiography is performed, the middle portion of LAD distal to the 1st diagonal branch is primarily chosen as the targeted occlusion location to induce MI [12] (Figure 2). In our experience, 60-minute mid-LAD occlusion is the most feasible location and timeframe to develop a porcine reperfused myocardial infarction model [12]. A commonly perceived difficulty of using the pig MI model is a predisposition for refractory arrhythmogenesis. Thus, it is known from previous reports using the porcine myocardial model, that there is a high incidence of ventricular fibrillation (VF) or ventricular tachycardia (VT) after induction of myocardial infarction coupled with greater mortality due to infarctrelated complications. All VF episodes occurred within the first 20-30 minutes after induction of myocardial infarction and within the first 10 minutes after reperfusion [12]. However, methods have been described to obviate this issue, such as aggressive airway protection and ventilatory management, electrolyte supplementation, and antiarrhythmic administration.

For histological assessment of injured myocardium, 2,3,5-Triphenyltetrazolium chloride (TTC) was commonly used. In viable myocardium, TTC is converted by dehydrogenases to a red formazan pigment that stains the tissue dark red. In necrotic myocardium, however, such staining does not occur because of the loss of dehydrogenases. To delineate areas at risk for ischemia and infarcted (necrotic) areas more clearly, our group performs in vivo a double staining method 
with $1 \%$ Evans blue dye and a $1 \%$ solution of TTC [12] (Figure 3).

2.2. Animal Models with Hibernating Myocardium. Myocardial hibernation, as originally described by Rahimtoola [13], was thought to represent "a state of persistently impaired myocardial and left ventricular function at rest because of reduced coronary blood flow that can be partially or completely restored to normal if the myocardial oxygen supply/demand relationship is favorably altered, either by improving blood flow or by reducing demand." Chronic reversible contractile dysfunction is frequently identified in the evaluation of patients with coronary artery disease. There has been considerable interest in prospectively identifying patients with chronic left ventricular contractile dysfunction that improves following coronary revascularization. PET and other noninvasive quantitative imaging modalities have advanced our understanding of the mechanisms involved with hibernating myocardium in patients [14-17]. From these advances, several studies were initiated to investigate suitable animal models. Usually, larger animals such as swine or dogs are used for developing a hibernating myocardium model. Initially, Matsuzaki et al. attempted to develop animal hibernation models through steady-state acute ischemia and short-term reduction in coronary flow reserve [18]. As a result, these models have provided insight into the initial adaptive response of the heart to acute ischemia, indicating that it can be maintained for several hours without the development of significant necrosis. Other groups have attempted to extend the duration of ischemia to 24 hours and 1 week for developing viable chronically dysfunctional myocardium [19-21]. Although these attempts resulted in regional dysfunction and a stenosis-induced reduction in coronary flow, one major drawback noted was that this approach resulted in irreversible injury that produced patchy necrosis visible by TTC in some animals and focal necrosis by light microscopy in most animals. These early animal experiments of short-term reductions in myocardial flow seemed to support this "smart" heart concept [22] in which myocardial function is downregulated to the point at which perfusion and function are once again in equilibrium. Chronic stenosis models that reduce coronary flow reserve appear to be more applicable to understanding factors governing the development of viable dysfunctional myocardium. Canty and Klocke have demonstrated that viable chronically dysfunctional myocardium could be reproduced in dogs [23], and Shen and Vatner have subsequently reproduced a similar state of chronic stunning using an Ameroid occcluder in the porcine model [24]. Fallavollita and Canty have further demonstrated that resting dysfunction worsened from 1 month to 2 months after operation despite resting perfusion at both time points being normal, consistent with a state of chronic stunning and hibernating myocardium with reduced resting coronary flow and increased FDG uptake developed at 3-4 months after operation [25]. Although the most widely used animal model of hibernating myocardium has been the Ameroid stenosis, the disadvantage of this approach is that both the rate and extent of progression of the Ameroid stenosis and the rate of collaterals are unpredictable. In addition, there is a large variation in the reported percent infarction of the area-at-risk (5 to $100 \%$ ). As a model of human coronary artery disease, this model has its limitations [19-21, 23-30].

\section{Experimental Porcine Coronary Model}

The coronary artery system of domestic pigs is in similar fashion to human coronary arteries $[31,32]$. Furthermore, when porcine coronary arteries are injured, thick neointima is seen within 28 days and is identical to human restenotic neointima (Figure 4). In addition, the amount of neointimal thickening is directly proportional to injury thereby permitting the creation of an injury-response regression relationship that can further quantify the response to potential treatment therapies [33, 34]. Cardiac catheterization techniques in the pig are similar to the techniques used in humans. Standard human diagnostic and interventional equipment can also be used. Thus, coronary arteries in domestic swine are suitable for the assessment of catheterbased interventional devices that may be used in humans.

3.1. The Evaluation of Stent Technologies. Drug-eluting stents (DES) have driven a new era into the field of percutaneous coronary intervention $[35,36]$. The success of this technology is founded not only on initial human clinical data but also on preclinical studies using the porcine coronary restenosis model [37-40]. Presently, it is unclear whether any single animal species is more predictive of the human response than another. These animal models can therefore help prove critical hypotheses regarding putative mechanisms of action of an intervention yet cannot be used to predict efficacy $[41,42]$. Regarding DES evaluation, recommendations from a preclinical studies consensus group suggest that the stent should be appropriately sized by visual or quantitative coronary artery measurement using a stent: artery ratio $\leq 1: 1$, while using a higher stent:artery ratio could induce severe arterial injury and considerable coronary artery stenosis $[41,42]$. For the evaluation of stent performance, a rigorous, (semi)quantitative and defined scale for device evaluation should be presented such as injury/inflammation score, vascular response/healing, and stent strut apposition/adjacent tissue as previously reported [43-48].

Although preclinical studies of both sirolimus-eluting stents (SESs) and paclitaxel-eluting stents (PESs) have demonstrated efficacy compared with bare metal stents (BMSs), enthusiasm for this technology has recently been dampened by concerns of late stent thrombosis. Nakazawa et al. has reported that incomplete endothelial coverage was seen in nonoverlapping and overlapping sites of both SES and PES compared with both the Endeavor zotarolimuseluting stent (ZES; Medtronic Vascular, Santa Rosa, CA) and BMS and that the differences were more pronounced in the overlapping segments [49] (Figure 5). In addition, two studies using human autopsy samples suggested that incomplete endothelial coverage of stent struts played an 


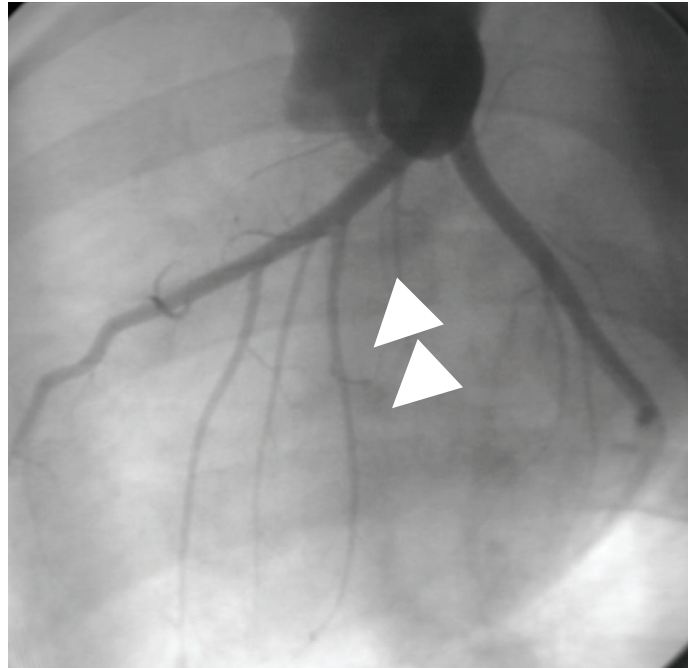

(a)

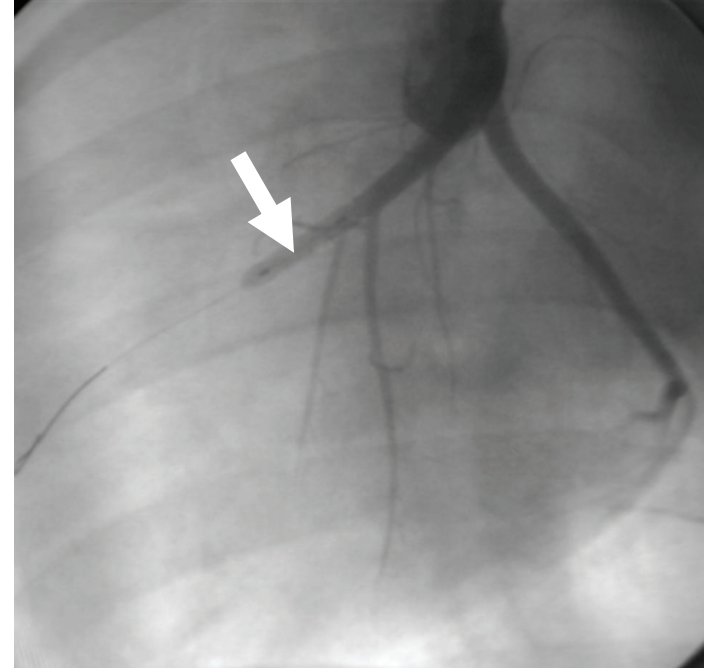

(b)

FIGURE 2: Baseline coronary angiogram was obtained for assessment of anatomic information of the LAD (a). The LAD with the first large diagonal artery (white arrowhead) is delineated. Angiographic confirmation of complete occlusion in the midportions (b) of the LAD with a PTCA balloon catheter (white arrow). Adapted from [12].

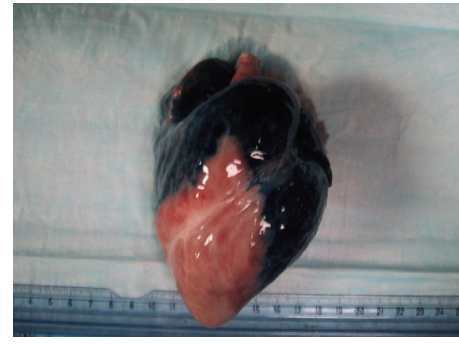

(a)

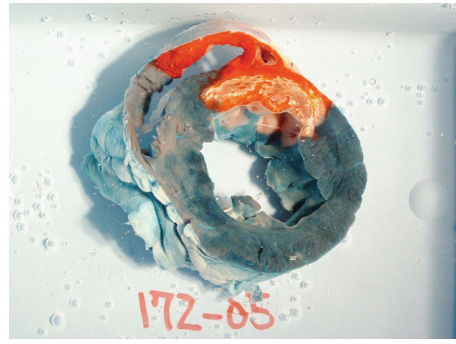

(b)
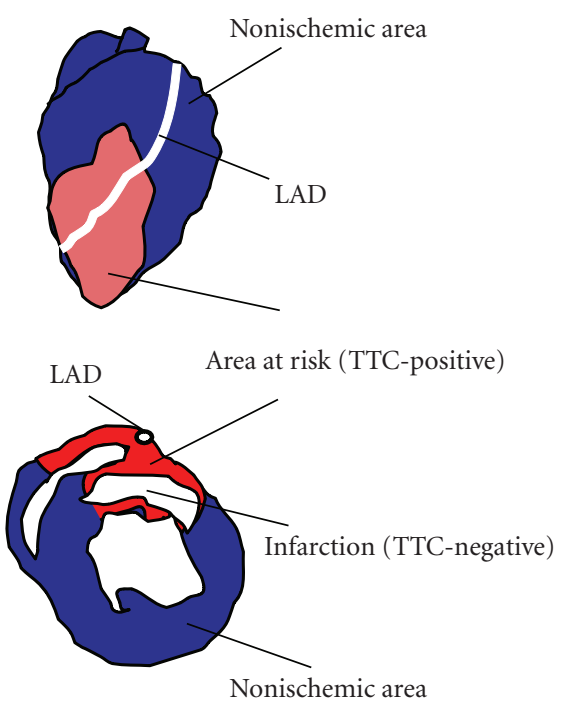

(c)

Figure 3: This picture demonstrates the porcine heart (a) and cross-sectional slice (b). The dark area stained with Evans Blue indicates nonischemic territory, and the red area stained with TTC is the area at risk for ischemia (TTC-positive). In (b), the white area is the infarcted (necrotic) myocardium (TTC-negative). The schema explains what the stained colors and territories indicate (c). LAD: left anterior descending artery. Adapted from [12].

important role as the morphometric predictor of late stent thrombosis $[52,53]$. From these reports, two time points are implicated for use in the evaluation of stent performance: the first at 28 days to observe for neointimal hyperplasia, and at least one later time point to examine long-term effects. The latter time point ( 3 or 6 months) depends on when "healing" and drug release are both complete. Of note, the FDA has typically recommended 6-month follow-up as the interval to acquire preclinical stent data $[41,42]$, yet this window reflects experience with balloon angioplasty and not necessarily the "window" for drug-eluting stents.

3.2. Porcine Heat-Injury Restenosis Model. The porcine coronary stent restenosis model is a well-accepted standard; however, its fundamental drawback is that the stent itself is 


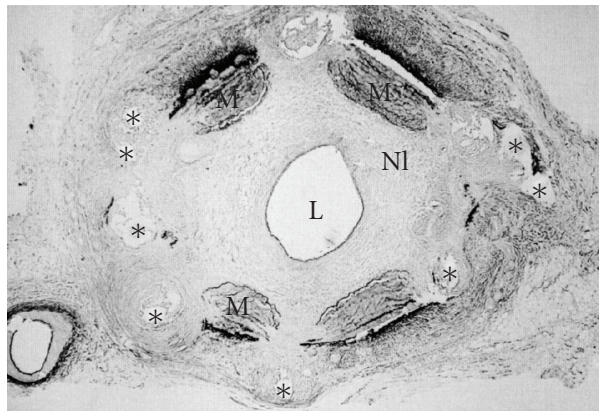

(a)

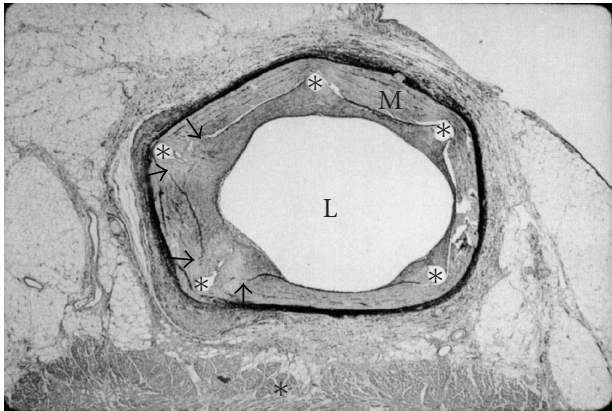

(b)

FIgURE 4: (a) Photomicrographic section shows gross neointimal proliferation causing a significant stenosis (Hematoxylin-Eosin stain, x5). (b) In this section, the porcine coronary was totally occluded with neointimal hyperplasia. L: lumen; M: media; NI: neointima; $*$ : stent strut. Adapted from the reference [32].
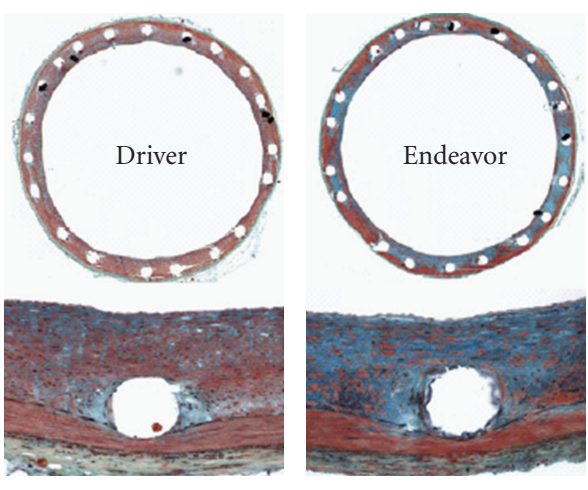
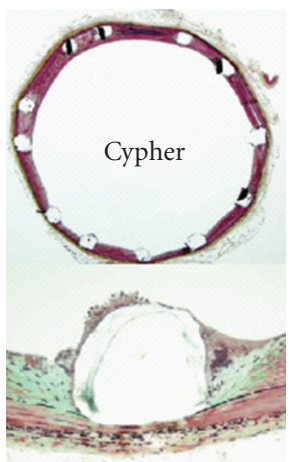
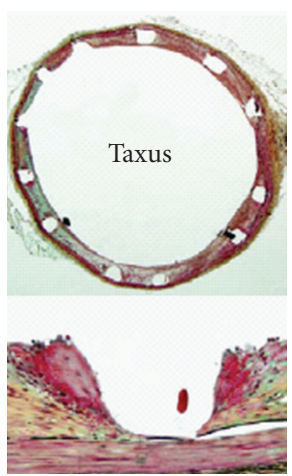

(a)

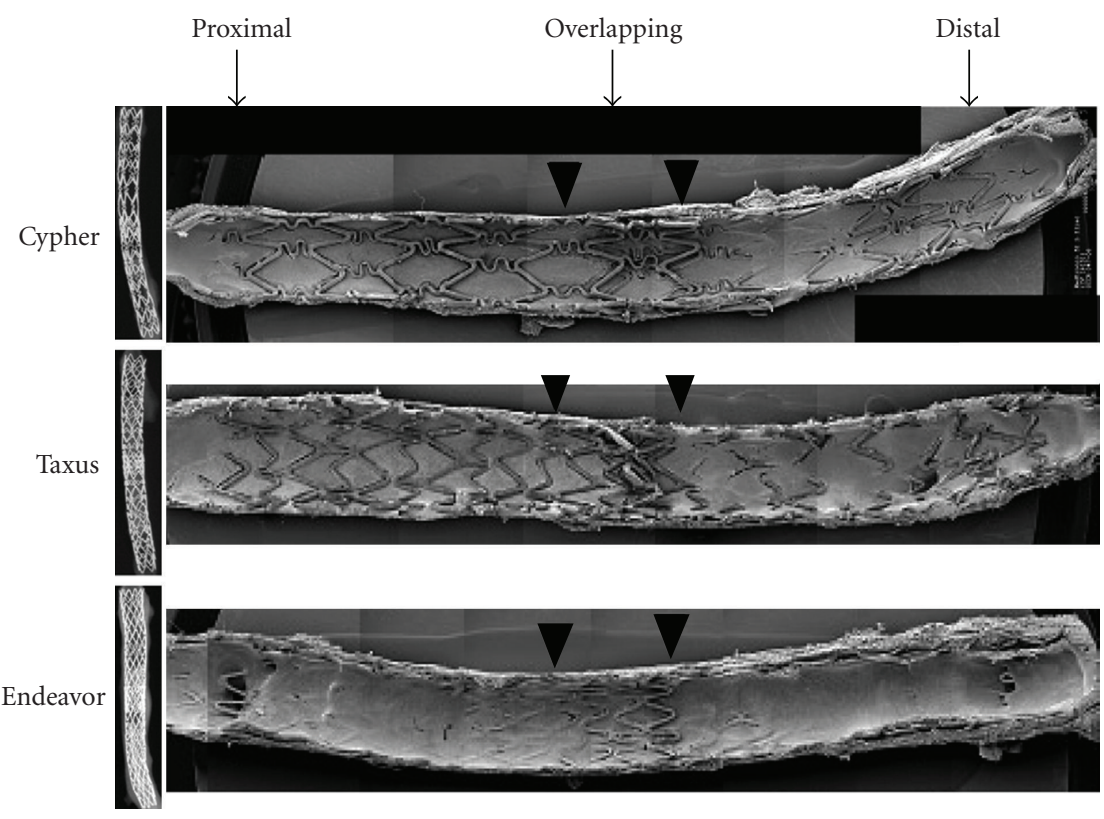

(b)

FIGURE 5: (a) X-rays of longitudinally cut rabbit iliac arteries at 21 days after placement of overlapping ZES, SES, and PES. The extent of stent coverage by endothelial cells was greatest with ZES, with almost complete coverage in the proximal and distal segments and significantly greater coverage in the overlapped segment compared with SES and PES. (b) Photomicrographs showing the amount of neointimal thickness at 28 days after placement of Endeavor zotarolimus-eluting stents (ZESs), Cypher sirolimus-eluting stents (SESs), Taxus paclitaxel-eluting stents (PESs), and Driver bare metal stents (BMSs) in rabbit iliac arteries. With SES, there were focally uncovered stent struts, which were associated with inflammation consisting of heterophils or eosinophils and giant cells. Adapted from [49]. 

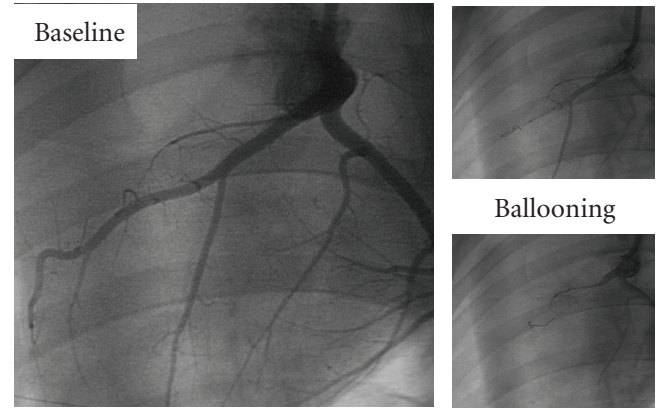

Ballooning
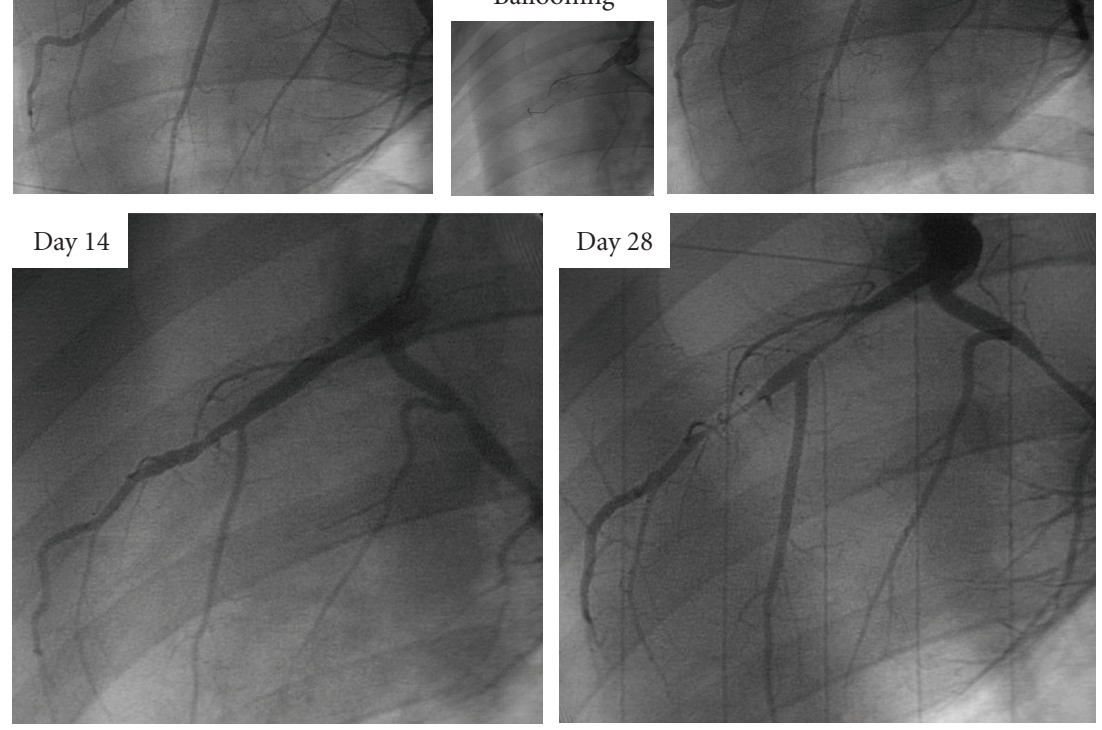

FIGURE 6: Time course of coronary artery treated with thermal balloon. A severe tandem coronary artery stenosis is observed in the left anterior descending artery (LAD) at 4 weeks after thermal balloon injury [50].

foreign material. As a result, this model may not be suitable to evaluate the performance of bifurcation or bioabsorbable stents due to lack of a true stenosis lesion. Also, results of coronary artery imaging such as computed tomography (CT), magnetic resonance imaging (MRI), intravascular ultrasound (IVUS), and optical coherence tomography (OCT) may be hampered as the stent can produce artifact. Using radiofrequency thermal balloon angioplasty, Staab et al. [54] and our laboratory [50] have investigated a porcine heat-injury restenosis model. In our study using 22 swine with a total of 54 coronary arteries, coronary artery stenoses were consistently developed at 4 weeks after heat-injury (Figure 6). In light of these results, this porcine coronary restenosis model might be useful for the evaluation of bifurcation stents and bioabsorbable stents, coronary imaging studies as listed above, and as part of the technical training for complex percutaneous coronary interventions such as bifurcation, diffuse lesion, and chronic total occlusion [50].

\subsection{Porcine Chronic Total Occlusion (CTO) Model. Recent} advances of DES technology have shifted focus within interventional cardiology from restenosis prevention to the treatment of CTO. Despite its common occurrence, there is surprisingly little information about the pathophysiology of CTO, and why some CTO can be crossed while in others, crossing is unsuccessful. For the past several years, researchers have developed CTO models to guide therapeutic investigations.
Spontaneous atherosclerotic plaque rupture and subsequent arterial occlusion do not occur naturally in any animal model, even among models genetically engineered to have increased atheroma formation. The initial method of producing a total occlusion utilized external ligature or Ameroid constriction [55]. However, one fundamental drawback of this method is the inability to facilitate the development of devices to recanalize CTO. Strauss et al. subsequently modified the thrombin injection model by infusing collagenase [56]. Several characteristics of human CTO were evident in this model, including mature fibrous tissue, multiple small intraluminal vascular channels, occasional extracellular lipid deposits, and disruption of the internal elastic lamina. Their reports suggested that the microchannels might be a critical determinant of successful CTO guide wire crossing [57]. Other CTO models have included stents with occluded outflow and have even used direct alcohol injection to promote thrombosis [58]. Developing an accurate and reproducible human-like coronary CTO model has been a complex undertaking because (1) coronary vessels are less amenable to a direct surgical approach; (2) simulating luminal and medial pathology, including microcalcification, has been difficult; (3) an inflammatory component must be present to mimic human CTO lesions $[59,60]$. Balloon angioplasty and stent implantation in animal coronary arteries, both standard methods of denuding the vessel and engendering neointimal proliferation, rarely result in CTO development. Polymers have also been used to invoke chronic coronary occlusions. Early polymeric implants were abandoned as stent platforms because they induced severe inflammatory responses and 


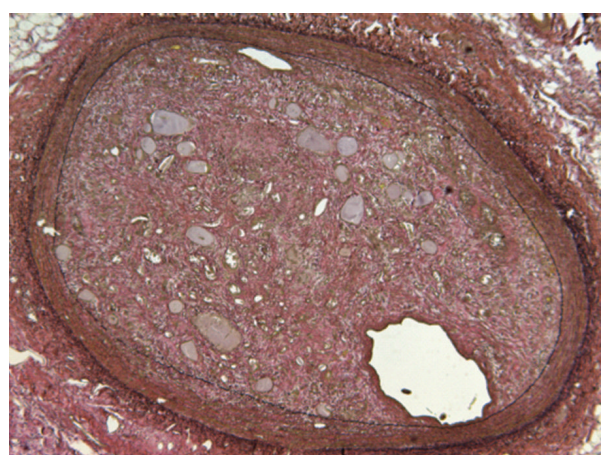

(a)

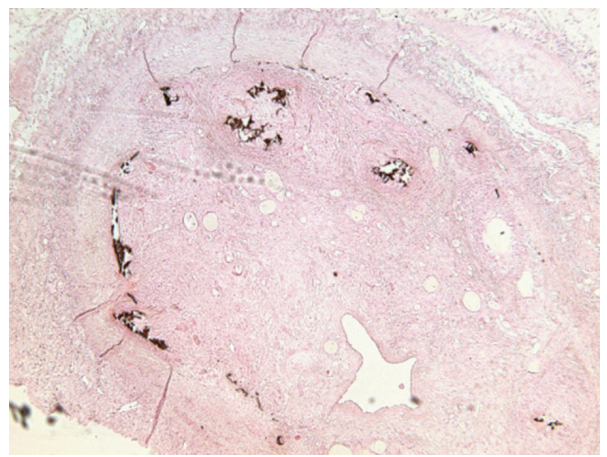

(c)

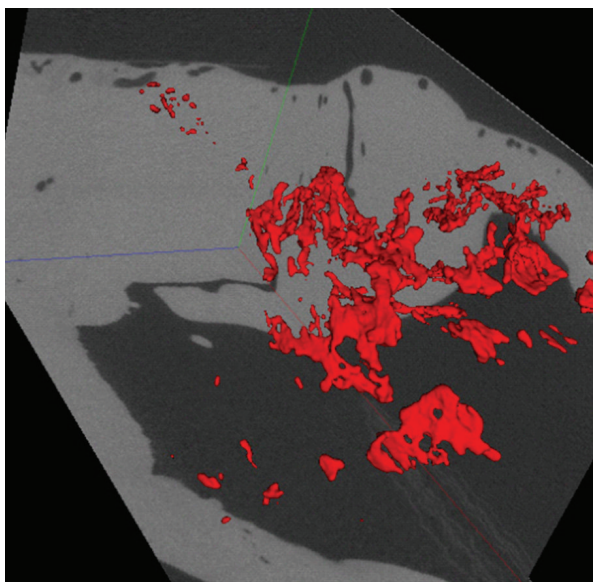

(e)

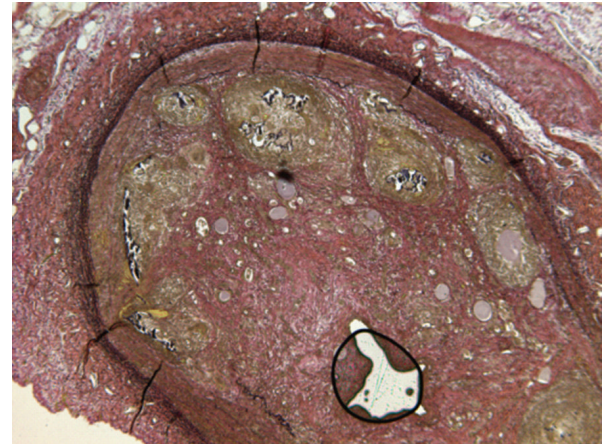

(b)

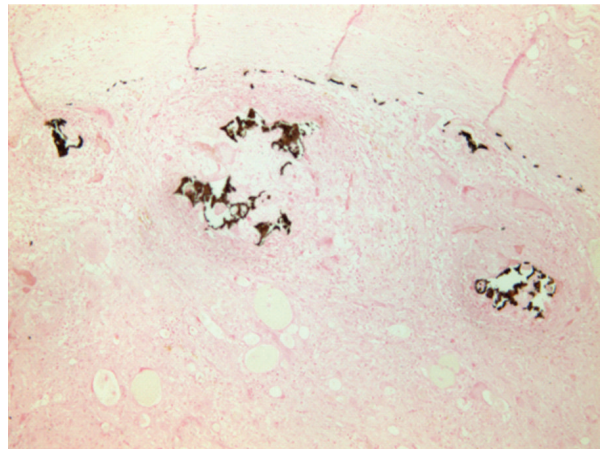

(d)

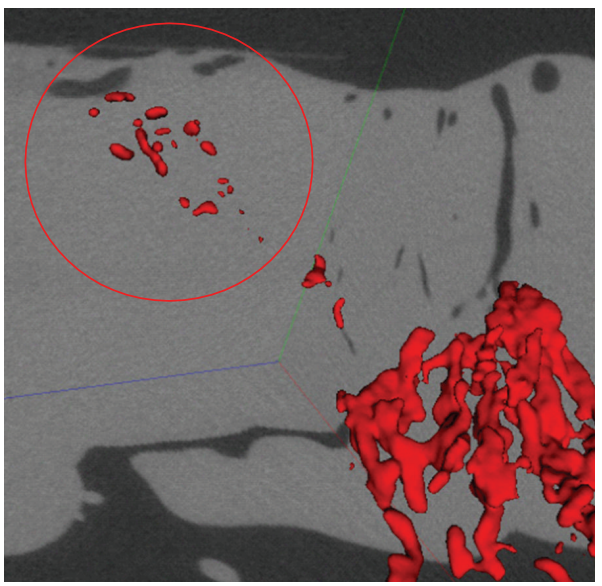

(f)

FIgURE 7: Histological examinations of pig experiments. Elastic-van Gieson (a, b) and Von kossa (c, d) stained CTO segment of coronary arteries. Arrows in (c) and (d) indicate microcalcification. Ex vivo micro-CT confirms that CTO lesions contain calcification in LAD (red circle) [51].

vessel occlusion [61]. Prosser et al. reported placement of a microporous poly L-lactic acid polymer into pig and dog coronary arteries [62]. The polymer is absorbed by 28 days, resulting in a microchanneled occlusion histologically similar to a human CTO. Using similar methods, Suzuki et al. [63] and our laboratory [51] have developed severe calcified CTO in pig coronary arteries (Figure 7). These animal models may contribute to a more in-depth understanding of the biology of human CTO and enable new device and pharmacological investigations to improve recanalization success in these challenging lesions.
3.4. Animal Model of Vulnerable Plaque. The definition of a "vulnerable" plaque is now accepted that most clinical manifestations of atherosclerosis such as AMI, unstable angina, and sudden cardiac death result from the development of an occlusive thrombus over an underlying plaque. The mechanisms of plaque rupture and subsequence occlusive thrombus formation are still unclear. Thus, animal models of high-risk (vulnerable) plaque are pivotal in the development and validation to identify and characterize vulnerable atherosclerotic lesions in humans. Recent models of plaque vulnerability continue to use small animals, particularly 
mice; however most do not result in features representative of human end-stage atherosclerosis. Characteristics of human vulnerable plaque such as plaque disruption, neovascularization, intraplaque hemorrhage, and occlusive thrombus formation occur rather infrequently in murine models. In addition, lipoprotein metabolism in mice is largely different from that in humans, thus a murine model may not be suitable for studies examining the effects of hypolipidemic therapies on atherosclerosis or myocardial infarction. In studies using large animals, Granada et al. reported that percutaneous intramural injection of cholesteryl linoleate results in the development of complex, lipid-containing inflammatory lesions in less than 4 weeks and that the intravascular ultrasound findings for the lesions created in this model demonstrate similar features to those of complex human atherosclerotic plaques $[64,65]$. However, this model is rich in smooth muscle cells/proteoglycans, and the lesions lack a necrotic core, calcification, and collagen (type I). Hasler-Rapacz et al. described a strain of large domestic swine which develop elevated LDL levels and subsequently develop human-like atherosclerotic lesions by 12 months of age $[66,67]$. Gerrity et al. also demonstrated the combination of induced diabetes by intravenous infusion of streptozotocin as well as hypercholesterolaemia by administration of a high cholesterol diet in Yorksire pigs, leading to advanced atherosclerotic lesions [68]. Although these porcine models develop human-like atherosclerosis, a major limitation is the time duration necessary for the development of advanced coronary lesions.

Shiomi et al. have developed the MI model, designated the Watanabe heritable hyperlipidemic (WHHL) rabbit, in which sudden cardiac events occur spontaneously without any artificial treatment [69]. In their study, the cumulative incidence of fatal sudden cardiac events up to the age of 35 months was $97 \%$, and representative findings of myocardial infarction (MI) such as vulnerable plaques as defined by Naghavi et al. [70] and thrombosis were observed in the hearts of those rabbits. This WHHL MI rabbit could be a very useful model for studying the mechanism(s) of plaque rupture and thrombogenesis and provide a novel means for developing new therapies or imaging technologies.

Currently, there is no standard animal model for vulnerable plaque. The availability of animal models of humanlike atherosclerosis will become the most critical element for the preclinical validation of emerging diagnostic/therapeutic technologies developed for use in patients with symptomatic coronary artery disease.

\section{Conclusions}

Clinical implementation of innovative diagnostic and therapeutic technologies will ultimately depend on the successful development of large animal models that permit preclinical evaluation of the technology. The field of percutaneous cardiovascular intervention technology is developing quickly and reflects the time sensitivity of the information contained within this paper. The success of this interventional subspecialty will be driven by the results of a collaborative relationship between the cardiologist, cardiac surgeon, and the medical device industry.

In addition, the role of animal models should be not only in device development and durability testing but also in training for clinicians in optimal techniques involving new procedures.

\section{Acknowledgment}

In the preparation of this paper, the authors thank Heidi Bonneau, RN, MS, for her editorial review.

\section{References}

[1] D. Bustad and D. Mcclellan, "Swine in biomedical research," Science, vol. 152, no. 3728, pp. 1526-1530, 1966.

[2] P. Verdouw D, M. A. Van Den Doel, S. De Zeeuw, and D. J. Duncker, "Animal models in the study of myocardial ischaemia and ischaemic syndromes," Cardiovascular Research, vol. 39, no. 1, pp. 121-135, 1998.

[3] J. Litvak, L. E. Siderides, and A. M. Vineberg, "The experimental production of coronary artery insufficiency and occlusion," American Heart Journal, vol. 53, no. 4, pp. 505-518, 1957.

[4] H. A. Fozzard, "Validity of myocardial infarction models," Circulation, vol. 52, no. 6, supplement, pp. 131-146, 1975.

[5] E. R. Schwarz, H. Montino, J. Fleischhauer, H. G. Klues, J. Vom Dahl, and P. Hanrath, "The angiotensin II receptor antagonist EXP 3174 reduces infarct size comparable with enalaprilat and augments preconditioning in the pig heart," Cardiovascular Drugs and Therapy, vol. 11, no. 5, pp. 687-695, 1997.

[6] E. R. Schwarz, J. Fleischhauer, H. Montino et al., "Infarct size reduction by ischemic preconditioning is a monophasic, short-lived phenomenon in anesthetized pigs," Journal of Cardiovascular Pharmacology and Therapeutics, vol. 3, no. 1, pp. 63-70, 1998.

[7] E. R. Schwarz, T. Reffelmann, F. Schoendube et al., "Hypoxic hypoperfusion fails to induce myocardial hibernation in anesthetized swine," Journal of Cardiovascular Pharmacology and Therapeutics, vol. 4, no. 4, pp. 235-247, 1999.

[8] D. L. Kraitchman, D. A. Bluemke, B. B. Chin, A. W. Heldman, and A. W. Heldman, "A minimally invasive method for creating coronary stenosis in a swine model for MRI and SPECT imaging," Investigative Radiology, vol. 35, no. 7, pp. 445-451, 2000.

[9] R. Edwards, Z. Yousef, R. Rakhit et al., "A model of closed chest regional myocardial infarction in the rabbit: a clinically relevant in vivo assay system of post-infarction remodelling," Basic Research in Cardiology, vol. 97, no. 5, pp. 374-383, 2002.

[10] T. Reffelmann, O. Sensebat, Y. Birnbaum et al., "A novel minimal-invasive model of chronic myocardial infarction in swine," Coronary Artery Disease, vol. 15, no. 1, pp. 7-12, 2004.

[11] G. A. Krombach, S. Kinzel, A. H. Mahnken, R. W. Günther, and A. Buecker, "Minimally invasive close-chest method for creating reperfused or occlusive myocardial infarction in swine," Investigative Radiology, vol. 40, no. 1, pp. 14-18, 2005.

[12] Y. Suzuki, J. K. Lyons, A. C. Yeung, and F. Ikeno, "In vivo porcine model of reperfused myocardial infarction: in situ double staining to measure precise infarct area/area at risk," Catheterization and Cardiovascular Interventions, vol. 71, no. 1, pp. 100-107, 2008.

[13] S. H. Rahimtoola, "A perspective on the three large multicenter randomized clinical trials of coronary bypass surgery for 
chronic stable angina," Circulation, vol. 72, no. 6, part 2, pp. V123-V135, 1985.

[14] C. Brunelli, O. Parodi, G. Sambuceti et al., "Improvement of hibernation in the clinical setting," Journal of Molecular and Cellular Cardiology, vol. 28, no. 12, pp. 2415-2418, 1996.

[15] J. Czernin, G. Porenta, R. Brunken et al., "Regional blood flow, oxidative metabolism, and glucose utilization in patients with recent myocardial infarction," Circulation, vol. 88, no. 3, pp. 884-895, 1993.

[16] P. Marzullo, O. Parodi, G. Sambuceti et al., "Residual coronary reserve identifies segmental viability in patients with wall motion abnormalities," Journal of the American College of Cardiology, vol. 26, no. 2, pp. 342-350, 1995.

[17] K. T. Sun, J. Czernin, J. Krivokapich et al., "Effects of dobutamine stimulation on myocardial blood flow, glucose metabolism, and wall motion in normal and dysfunctional myocardium," Circulation, vol. 94, no. 12, pp. 3146-3154, 1996.

[18] M. Matsuzaki, K. P. Gallagher, and W. S. Kemper, "Sustained regional dysfunction produced by prolonged coronary stenosis: gradual recovery after reperfusion," Circulation, vol. 68, no. 1, pp. 170-182, 1983.

[19] C. Chen, L. Chen, J. T. Fallon et al., "Functional and structural alterations with 24-hour myocardial hibernation and recovery after reperfusion: a pig model of myocardial hibernation," Circulation, vol. 94, no. 3, pp. 507-516, 1996.

[20] C. Chen, L. Ma, W. Dyckman et al., "Left ventricular remodeling in myocardial hibernation," Circulation, vol. 96, no. 9, supplement, pp. II46-II50, 1997.

[21] R. K. Kudej, B. Ghaleh, N. Sato, Y. T. Shen, S. P. Bishop, and S. F. Vatner, "Ineffective perfusion-contraction matching in conscious, chronically instrumented pigs with an extended period of coronary stenosis," Circulation Research, vol. 82, no. 11, pp. 1199-1205, 1998.

[22] S. H. Rahimtoola, "The hibernating myocardium," American Heart Journal, vol. 117, no. 1, pp. 211-221, 1989.

[23] J. M. Canty and F. J. Klocke, "Reductions in regional myocardial function at rest in conscious dogs with chronically reduced regional coronary artery pressure," Circulation Research, vol. 61, no. 5, part 2, pp. I-107-I-116, 1987.

[24] Y. T. Shen and S. F. Vatner, "Mechanism of impaired myocardial function during progressive coronary stenosis in conscious pigs: hibernation versus stunning?" Circulation Research, vol. 76, no. 3, pp. 479-488, 1995.

[25] J. A. Fallavollita and J. M. Canty, "Differential F-2deoxyglucose uptake in viable dysfunctional myocardium with normal resting perfusion: evidence for chronic stunning in pigs," Circulation, vol. 99, no. 21, pp. 2798-2805, 1999.

[26] A. J. Liedtke, B. Renstrom, S. H. Nellis, J. L. Hall, and W. C. Stanley, "Mechanical and metabolic functions in pig hearts after 4 days of chronic coronary stenosis," Journal of the American College of Cardiology, vol. 26, no. 3, pp. 815-825, 1995.

[27] S. Firoozan, K. Wei, A. Linka, D. Skyba, N. C. Goodman, and S. Kaul, "A canine model of chronic ischemic cardiomyopathy: characterization of regional flow-function relations," American Journal of Physiology, vol. 276, no. 2, part 2, pp. H446H455, 1999.

[28] I. Mills, J. T. Fallon, D. Wrenn et al., "Adaptive responses of coronary circulation and myocardium to chronic reduction in perfusion pressure and flow," American Journal of Physiology, vol. 266, no. 2, part 2, pp. H447-H457, 1994.
[29] J. A. Fallavollita, B. J. Perry, and J. M. Canty, "F-2Deoxyglucose deposition and regional flow in pigs with chronically dysfunctional myocardium: evidence for transmural variations in chronic hibernating myocardium," Circulation, vol. 95, no. 7, pp. 1900-1909, 1997.

[30] E. O. McFalls, D. Baldwin, B. Palmer et al., "Regional glucose uptake within hypoperfused swine myocardium as measured by positron emission tomography," American Journal of Physiology, vol. 272, no. 1, part 2, pp. H343-H349, 1997.

[31] R. S. Schwartz, J. G. Murphy, W. D. Edwards, A. R. Camrud, R. E. Vlietstra, and D. R. Holmes, "Restenosis after balloon angioplasty. A practical proliferative model in porcine coronary arteries," Circulation, vol. 82, no. 6, pp. 2190-2200, 1990.

[32] R. S. Schwartz, K. C. Huber, J. G. Murphy et al., "Restenosis and the proportional neointimal response to coronary artery injury: results in a porcine model," Journal of the American College of Cardiology, vol. 19, no. 2, pp. 267-274, 1992.

[33] R. S. Schwartz, D. J. Holder, D. R. Holmes et al., "Neointimal thickening after severe coronary artery injury is limited by short-term administration of a factor Xa inhibitor: results in a porcine model," Circulation, vol. 93, no. 8, pp. 1542-1548, 1996.

[34] K. C. Huber, R. S. Schwartz, W. D. Edwards et al., "Effects of angiotensin converting enzyme inhibition on neointimal proliferation in a porcine coronary injury model," American Heart Journal, vol. 125, no. 3, pp. 695-701, 1993.

[35] P. W. Serruys, M. Degertekin, K. Tanabe et al., "Intravascular ultrasound findings in the multicenter, randomized, doubleblind RAVEL (RAndomized study with the sirolimus-eluting VElocity balloon-expandable stent in the treatment of patients with de novo native coronary artery lesions) trial," Circulation, vol. 106, no. 7, pp. 798-803, 2002.

[36] J. E. Sousa, M. A. Costa, A. Abizaid et al., "Sirolimus-eluting stent for the treatment of in-stent restenosis: a quantitative coronary angiography and three-dimensional intravascular ultrasound study," Circulation, vol. 107, no. 1, pp. 24-27, 2003.

[37] R. Gallo, A. Padurean, T. Jayaraman et al., "Inhibition of intimal thickening after balloon angioplasty in porcine coronary arteries by targeting regulators of the cell cycle," Circulation, vol. 99, no. 16, pp. 2164-2170, 1999.

[38] T. Suzuki, G. Kopia, S. I. Hayashi et al., "Stent-based delivery of sirolimus reduces neointimal formation in a porcine coronary model," Circulation, vol. 104, no. 10, pp. 1188-1193, 2001.

[39] P. S. Teirstein, "Living the dream of no restenosis," Circulation, vol. 104, no. 17, pp. 1996-1998, 2001.

[40] A. W. Heldman, L. Cheng, G. M. Jenkins et al., "Paclitaxel stent coating inhibits neointimal hyperplasia at 4 weeks in a porcine model of coronary restenosis," Circulation, vol. 103, no. 18, pp. 2289-2295, 2001.

[41] R. S. Schwartz, E. R. Edelman, A. Carter et al., "Drug-eluting stents in preclinical studies recommended evaluation from a consensus group," Circulation, vol. 106, no. 14, pp. 1867-1873, 2002.

[42] R. S. Schwartz, E. Edelman, R. Virmani et al., "Drug-eluting stents in preclinical studies: updated consensus recommendations for preclinical evaluation," Circulation, vol. 1, no. 2, pp. 143-153, 2008.

[43] R. S. Schwartz, D. R. Holmes, and E. J. Topol, "The restenosis paradigm revisites: an alternative proposal for cellular mechanisms," Journal of the American College of Cardiology, vol. 20, no. 5, pp. 1284-1293, 1992.

[44] R. Kornowski, M. K. Hong, F. O. Tio, O. Bramwell, H. $\mathrm{Wu}$, and M. B. Leon, "In-stent restenosis: contributions of inflammatory responses and arterial injury to neointimal 
hyperplasia," Journal of the American College of Cardiology, vol. 31, no. 1, pp. 224-230, 1998.

[45] A. J. Taylor, P. D. Gorman, B. Kenwood, C. Hudak, G. Tashko, and R. Virmani, "A comparison of four stent designs on arterial injury, cellular proliferation, neointima formation, and arterial dimensions in an experimental porcine model," Catheterization and Cardiovascular Interventions, vol. 53, no. 3, pp. 420-425, 2001.

[46] A. J. Carter, J. R. Laird, A. Farb, W. Kufs, D. C. Wortham, and R. Virmani, "Morphologic characteristics of lesion formation and time course of smooth muscle cell proliferation in a porcine proliferative restenosis model," Journal of the American College of Cardiology, vol. 24, no. 5, pp. 1398-1405, 1994.

[47] E. R. Edelman and C. Rogers, "Pathobiologic responses to stenting," American Journal of Cardiology, vol. 81, no. 7 A, pp. 4E-6E, 1998.

[48] R. Virmani, F. D. Kolodgie, A. Farb, and A. Lafont, "Drug eluting stents: are human and animal studies comparable?" Heart, vol. 89, no. 2, pp. 133-138, 2003.

[49] G. Nakazawa, A. V. Finn, M. C. John, F. D. Kolodgie, and R. Virmani, "The significance of preclinical evaluation of sirolimus-, paclitaxel-, and zotarolimus-eluting stents," American Journal of Cardiology, vol. 100, no. 8B, pp. M36M44, 2007.

[50] Y. Suzuki, J. K. Lyons, A. C. Yeung, and F. Ikeno, “The porcine restenosis model using thermal balloon injury: comparison with the model by coronary stenting," Journal of Invasive Cardiology, vol. 20, no. 3, pp. 142-146, 2008.

[51] Y. Suzuki, A. Oyane, F. Ikeno, J. K. Lyons, and A. C. Yeung, "Development of animal model for calcified chronic total occlusion," Catheterization and Cardiovascular Interventions, vol. 74, no. 3, pp. 468-475, 2009.

[52] A. V. Finn, F. D. Kolodgie, J. Harnek et al., "Differential response of delayed healing and persistent inflammation at sites of overlapping sirolimus- or paclitaxel-eluting stents," Circulation, vol. 112, no. 2, pp. 270-278, 2005.

[53] M. Joner, A. V. Finn, A. Farb et al., "Pathology of drug-eluting stents in humans: delayed healing and late thrombotic risk," Journal of the American College of Cardiology, vol. 48, no. 1, pp. 193-202, 2006.

[54] M. E. Staab, S. S. Srivatsa, A. Lerman et al., "Arterial remodeling after experimental percutaneous injury is highly dependent on adventitial injury and histopathology," International Journal of Cardiology, vol. 58, no. 1, pp. 31-40, 1997.

[55] W. E. Elzinga, "Ameroid constrictor: uniform closure rates and a calibration procedure," Journal of Applied Physiology, vol. 27, no. 3, pp. 419-421, 1969.

[56] B. H. Strauss, L. Goldman, B. Qiang et al., "Collagenase plaque digestion for facilitating guide wire crossing in chronic total occlusions," Circulation, vol. 108, no. 10, pp. 1259-1262, 2003.

[57] B. H. Strauss, A. Segev, G. A. Wright et al., "Microvessels in chronic total occlusions: pathways for successful guidewire crossing?" Journal of Interventional Cardiology, vol. 18, no. 6, pp. 425-436, 2005.

[58] S. Nikol, S. Armeanu, M. G. Engelmann et al., "Evaluation of endovascular techniques for creating a porcine femoral artery occlusion model," Journal of Endovascular Therapy, vol. 8, no. 4, pp. 401-407, 2001.

[59] M. Katsuragawa, H. Fujiwara, M. Miyamae, and S. Sasayama, "Histologic studies in percutaneous transluminal coronary angioplasty for chronic total occlusion: comparison of tapering and abrupt types of occlusion and short and long occluded segments," Journal of the American College of Cardiology, vol. 21, no. 3, pp. 604-611, 1993.

[60] S. S. Srivatsa, W. D. Edwards, C. M. Boos et al., "Histologic correlates of angiographic chronic total coronary artery occlusions. Influence of occlusion duration on neovascular channel patterns and intimal plaque composition," Journal of the American College of Cardiology, vol. 29, no. 5, pp. 955-963, 1997.

[61] J. F. Tanguay, J. P. Zidar, H. R. Phillips, and R. S. Stack, "Current status of biodegradable stents," Cardiology Clinics, vol. 12, no. 4, pp. 699-713, 1994.

[62] L. Prosser, C. M. Agrawal, J. Polan, J. Elliott, D. G. Adams, and S. R. Bailey, "Implantation of oxygen enhanced, threedimensional microporous L-PLA polymers: a reproducible porcine model of chronic total coronary occlusion," Catheterization and Cardiovascular Interventions, vol. 67, no. 3, pp. 412416, 2006.

[63] K. Suzuki, N. Saito, G. Zhang et al., "Development of a novel calcified total occlusion model in porcine coronary arteries," Journal of Invasive Cardiology, vol. 20, no. 6, pp. 296-301, 2008.

[64] J. F. Granada, P. R. Moreno, A. P. Burke, D. G. Schulz, A. E. Raizner, and G. L. Kaluza, "Endovascular needle injection of cholesteryl linoleate into the arterial wall produces complex vascular lesions identifiable by intravascular ultrasound: early development in a porcine model of vulnerable plaque," Coronary Artery Disease, vol. 16, no. 4, pp. 217-224, 2005.

[65] J. F. Granada, D. Wallace-Bradley, H. K. Win et al., "In vivo plaque characterization using intravascular ultrasound-virtual histology in a porcine model of complex coronary lesions," Arteriosclerosis, Thrombosis, and Vascular Biology, vol. 27, no. 2, pp. 387-393, 2007.

[66] J. Hasler-Rapacz, H. Ellegren, A. K. Fridolfsson et al., "Identification of a mutation in the low density lipoprotein receptor gene associated with recessive familial hypercholesterolemia in swine," American Journal of Medical Genetics, vol. 76, no. 5, pp. 379-386, 1998.

[67] M. F. Prescott, C. H. McBride, J. Hasler-Rapacz, J. Von Linden, and J. Rapacz, "Development of complex atherosclerotic lesions in pigs with inherited hyper-LDL cholesterolemia bearing mutant alleles for apolipoprotein B," American Journal of Pathology, vol. 139, no. 1, pp. 139-147, 1991.

[68] R. G. Gerrity, R. Natarajan, J. L. Nadler, and T. Kimsey, "Diabetes-Induced Accelerated Atherosclerosis in Swine," Diabetes, vol. 50, no. 7, pp. 1654-1665, 2001.

[69] M. Shiomi, T. Ito, S. Yamada, S. Kawashima, and J. Fan, "Correlation of vulnerable coronary plaques to sudden cardiac events. Lessons from a myocardial infarction-prone animal model (the WHHLMI rabbit)," Journal of Atherosclerosis and Thrombosis, vol. 11, no. 4, pp. 184-189, 2004.

[70] M. Naghavi, P. Libby, E. Falk et al., "From vulnerable plaque to vulnerable patient: a call for new definitions and risk assessment strategies: part II," Circulation, vol. 108, no. 15, pp. 1772-1778, 2003. 


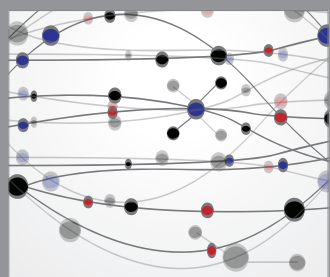

The Scientific World Journal
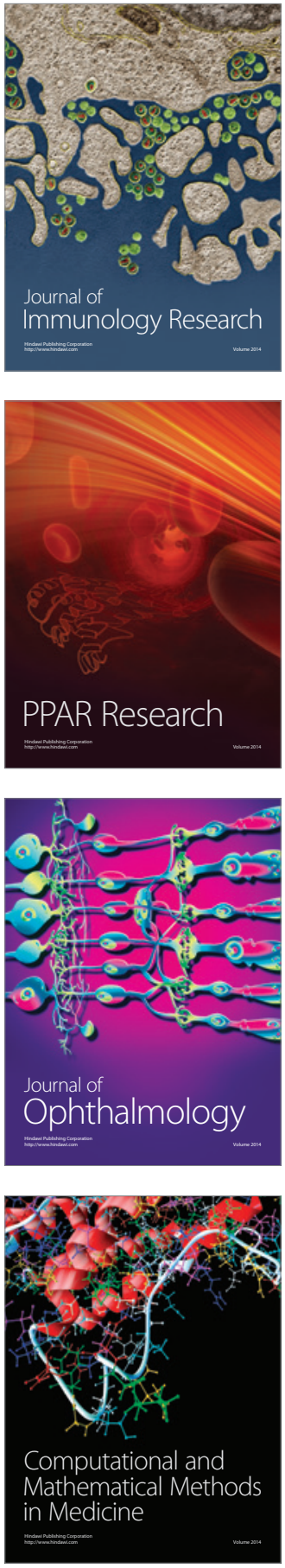

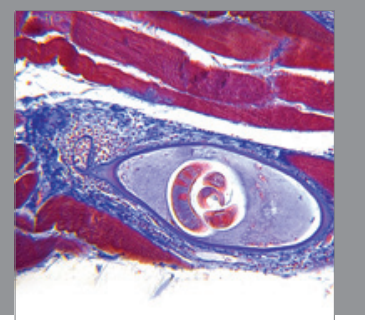

Gastroenterology

Research and Practice
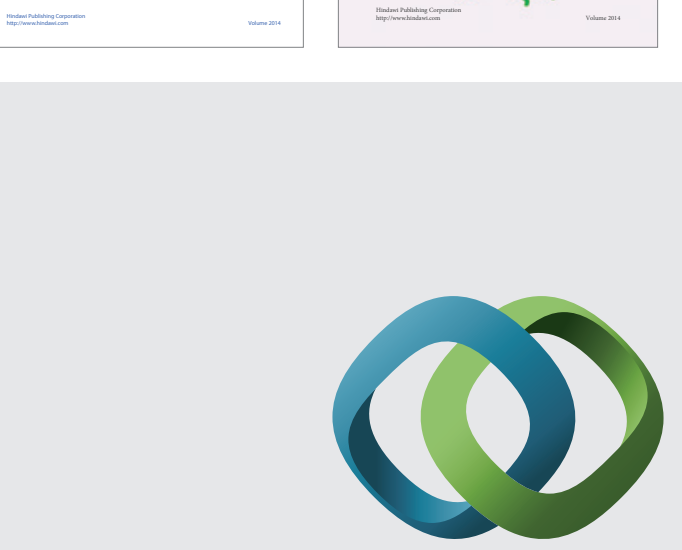

\section{Hindawi}

Submit your manuscripts at

http://www.hindawi.com
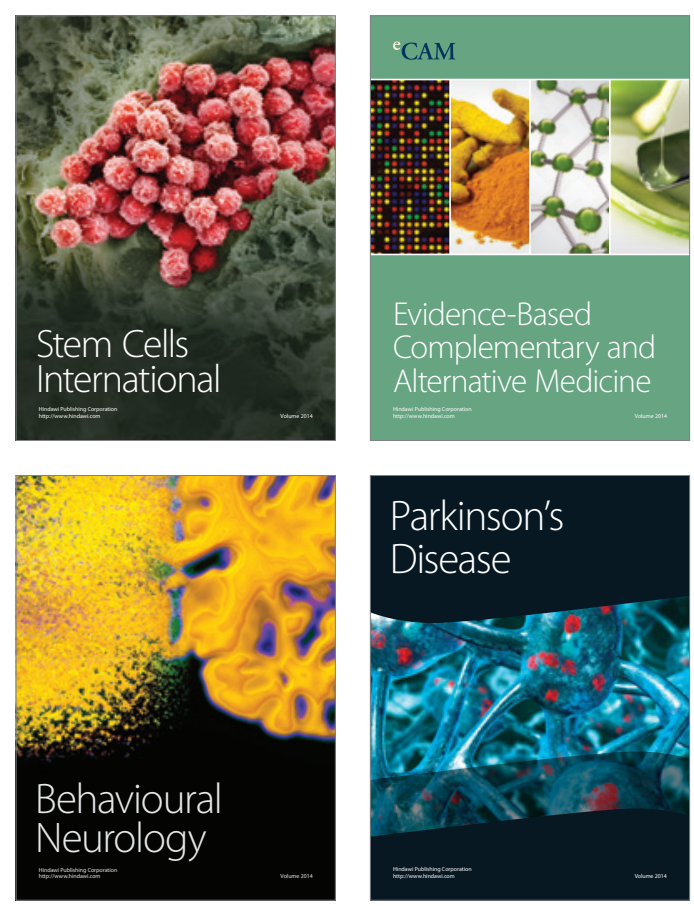

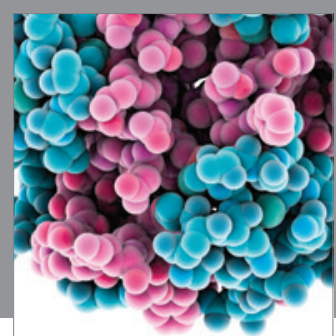

Journal of
Diabetes Research

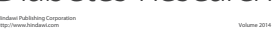

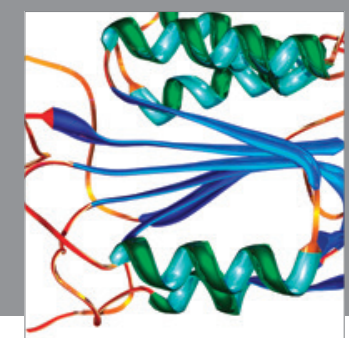

Disease Markers
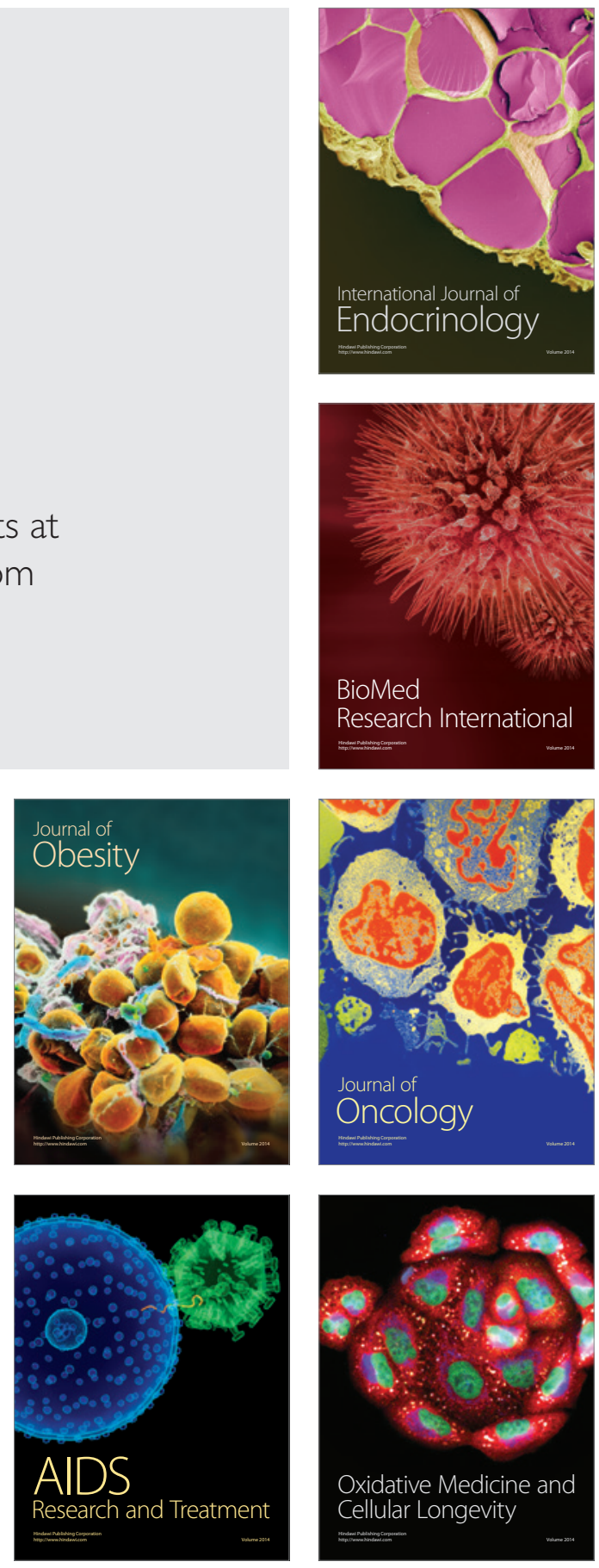